\title{
Modifying the multi-arm multi-stage (MAMS) design for use in a phase II tuberculosis trial in sub-Saharan Africa with a time-to-event primary outcome
}

\author{
Patrick Phillips ${ }^{4 *}$, Michael Hoelscher ${ }^{2}$, Daniel Bratton ${ }^{4}$, Sunita Rehal ${ }^{4}$, Norbert Heinrich², \\ Georgette Plemper van Balen ${ }^{1}$, Andrew Nunn ${ }^{4}$, Rob Aarnoutse', Sonja Henne², Stephen Gillespie ${ }^{3}$, Martin Boeree', \\ PanACEA consortium
}

From 2nd Clinical Trials Methodology Conference: Methodology Matters

Edinburgh, UK. 18-19 November 2013

\section{Background}

Tuberculosis (TB) causes significant morbidity and mortality globally. High efficacy in clinical trials of the current 4-drug 6-month regimen does not always translate into practice due to a heavy pill burden and long duration. New drugs are in late-phase clinical development, so trials that evaluate novel and existing drugs in combination are urgently needed. A multi-arm multi-stage (MAMS) design, with planned interim analyses allowing stopping of arms not showing benefit, has been developed and used in oncology. We have adapted this approach to create the PanACEA MAMS-TB 5-arm 3-stage phase II trial evaluating new treatments for $\mathrm{TB}$.

\section{Methods}

The transition from an endpoint of progression-free survival to one of negative culture conversion required several modifications including allowing for fixed follow-up of 12 weeks and targeting an increase in the hazard of the event rather than a decrease. The Weibull distribution was used in place of the exponential distribution to model a more appropriate increasing hazard. The option of a second interim analysis protects against slow recruitment.

\section{Results}

In this paper, the modifications to the design will be explained with discussion around pair-wise and family- wise type I error rates, power and expected sample size in comparison with a fixed sample design.

\section{Conclusions}

Reductions in expected sample size are moderate in this relatively small 5 -arm study due to the absence of a realtime biomarker. Nevertheless, there is potential for faster evaluation of more regimens in a larger phase II/III design once better biomarkers are validated.

\section{Authors' details}

${ }^{1}$ Radboud University Nijmegen Medical Centre, Nijmegen, The Netherlands, ${ }^{2}$ Medical Centre of the University of Munich (LMU), Munich, Germany.

${ }^{3}$ University of St Andrews School of Medicine, St. Andrews, UK. ${ }^{4}$ MRC Clinical Trials Unit, London, UK.

Published: 29 November 2013

doi:10.1186/1745-6215-14-S1-P26

Cite this article as: Phillips et al:: Modifying the multi-arm multi-stage (MAMS) design for use in a phase II tuberculosis trial in sub-Saharan Africa with a time-to-event primary outcome. Trials 2013 14(Suppl 1):P26.

${ }^{4}$ MRC Clinical Trials Unit, London, UK

Full list of author information is available at the end of the article

(c) 2013 Phillips et al; licensee BioMed Central Ltd. This is an Open Access article distributed under the terms of the Creative Commons 